\title{
Capsule Commentary on Bolton et al., Integrating Personalized Care Planning into Primary Care: a Multiple-Case Study of Early Adopting Patient-Centered Medical Homes
}

Parker Magin, PhD, FRACGP

Discipline of General Practice, School of Medicine and Public Health , University of Newcastle, Callaghan, Australia.

J Gen Intern Med 35(2):621

DOI: $10.1007 / \mathrm{s} 11606-019-05577-4$

(c) Society of General Internal Medicine 2019

$\mathrm{P}$ atient-centered care is essential to chronic disease management and to management of multimorbidity. ${ }^{1,2}$ Personalized care planning represents a systematic approach to patient-centered management of patients with chronic and comorbid conditions. ${ }^{3,4}$

Bolton et al..$^{5}$ examine the operation of a US Veterans Health Administration (VHA)-initiated version of personalized care planning (personal health planning (PHP)). Personalized care planning, involving holistic care incorporating patient values, preferences, and expectations within a collaborative and iterative team-based process, is not straightforward. Bolton et al.'s objective (to examine the implementation of PHP in four VHA patient-centered medical home clinics) was challenging and they adopted a research methodology appropriate to the complexity of PHP: a qualitative multiple case study design with triangulation of several data sources for each case. This methodology has provided a rich exploration of the topic with findings that are of importance in considering personalized care planning in primary care and the organizational and system factors that underpin its integration into practice. Unsurprisingly, the investigators found that personalized care planning is complicated to implement.

Also unsurprisingly, given this complexity of implementation, Bolton et al. found markedly heterogeneous approaches to PHP in the four centers studied. From this heterogeneity, they identified a number of specific program components critical to successful implementation: planning beyond the initial assessment of patient priorities; framing the initiative for patients; a team-based approach to care plan development and delivery; not depending solely on the electronic health record for communication; and engaging stakeholders in framing the model of implementation to be pursued.

Published online December 10, 2019
An overall reading of Bolton et al.'s findings is that the vital overarching theme is communication (and that this communication needs to be active rather than wholly Electronic Medical Record-dependent). Active communication between patient, primary physician, and other clinical and non-clinical team members underpins the formulation of, and on-theground delivery and iteration of, individual PHP. It informs flexibility of individual patient plans. At a systems level, stakeholder communication is also vital in achieving commitment to and investment in a PHP program structure that will be acceptable, practicable, and productive in a particular local context.

Corresponding Author: Parker Magin, PhD, FRACGP; Discipline of General Practice, School of Medicine and Public Health University of Newcastle, Callaghan 2308, Australia (e-mail: parker. magin@newcastle.edu.au).

\section{Compliance with ethical standards:}

Conflict of interest: The authors declare that they do not have a conflict of interest.

\section{REFERENCES}

1. National Institute for Health and Care Excellence. Multimorbidity. Clinical assessment and management. NG56. 2016. https://www.nice.org.uk/ guidance/ng56. (Accessed 4/11/19)

2. Rosland AM, Wong E, Maciejewski M, et al. Patient-centered medical home implementation and improved chronic disease quality: a longitudinal observational study. Health Serv Res 2018;53:2503-22.

3. Patient-centered Primary Care Collaborative. Defining the medical home. A patient-centered philosophy that drives primary care excellence. https:// www.pcpcc.org/about/medical-home(Accessed 4/11/19)

4. Edwards ST. Dorr DA, Landon BE. Can personalized care planning improve primary care? JAMA. 2017;318:25-6.

5. Bolton R, Bokhour B, Hogan T, Luger T, Ruben M, Fix G. Integrating personalized care planning into primary care: a multiple-case study of early adopting patient-centered medical homes. J Gen Intern Med. https://doi.org/10.1007/s11606-019-05418-4.

Publisher's Note Springer Nature remains neutral with regard to jurisdictional claims in published maps and institutional affiliations. 